\title{
HIGH QUALITY RECONSTRUCTION OF DYNAMIC OBJECTS USING 2D-3D CAMERA FUSION
}

\author{
Cansen Jiang ${ }^{1}$, Dennis Christie ${ }^{2}$, Danda Pani Paudel ${ }^{3}$, and Cédric Demonceaux ${ }^{1}$ \\ 1. Le2i, FRE CNRS 2005, Arts et Métiers, Univ. Bourgogne Franche-Comté, France. \\ 2. Gunadarma University, Indonesia. \\ 3. Computer Vision Lab, ETH Zürich, Switzerland.
}

\begin{abstract}
In this paper, we propose a complete pipeline for high quality reconstruction of dynamic objects using 2D-3D camera setup attached to a moving vehicle. Starting from the segmented motion trajectories of individual objects, we compute their precise motion parameters, register multiple sparse point clouds to increase the density, and develop a smooth and textured surface from the dense (but scattered) point cloud. The success of our method relies on the proposed optimization framework for accurate motion estimation between two sparse point clouds. Our formulation for fusing closest-point and consensus based motion estimations, respectively in the absence and presence of motion trajectories, is the key to obtain such accuracy. Several experiments performed on both synthetic and real (KITTI) datasets show that the proposed framework is very robust and accurate.
\end{abstract}

Index Terms - 3D Reconstruction, 2D-3D Fusion, Point Cloud Registration, RANSAC, ICP

\section{INTRODUCTION}

Scene reconstruction and modelling are two major tasks of 3D Computer Vision. The reconstruction offers us the exact observation of the 3-dimensional world, whereas, modelling allows us to perceive it accurately. Both of these tasks have always been active areas of research due to their wide range of potential applications, such as scene representation, understanding, and robot navigation [1].

For a moving 2D-3D camera setup, the $3 \mathrm{D}$ reconstruction of the scene can be obtained by registering a sequence of point clouds with the help of Visual Odometry (VO) measurements [2,3]. However, the VO-based registration is valid only for the static scene parts. Therefore, such reconstruction suffers from several visual artefacts due to the dynamic parts. In this regard, recent work by Jiang et al. [4-6] categorizes the scene into static and dynamic parts before performing VO. Their method focuses on improving VO measurements, and the attempted dynamic object reconstruction is rather preliminary and naive. In this work, we focus on the high quality

This research has been funded in part by ANR (Agence Nationale de la Recherche-France) International Project pLaTINUM (ANR-15-CE23-0010).

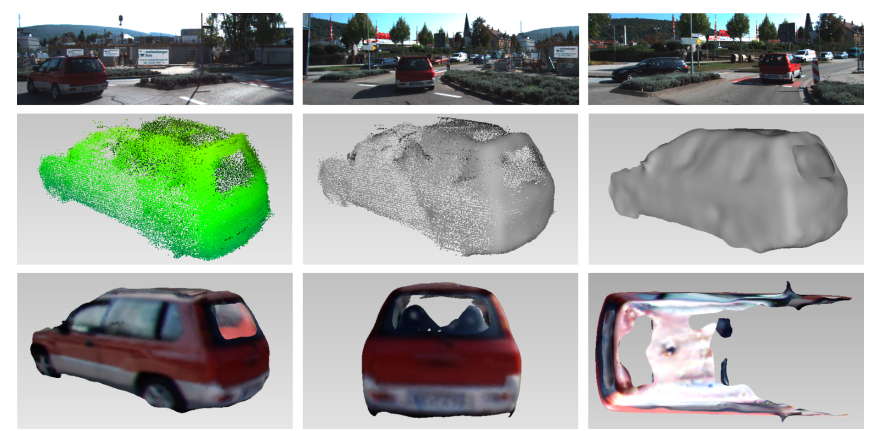

Fig. 1: Moving Car Reconstruction from a Mobile Platform: Top are selected frames of a moving car. Middle show the registered sparse point cloud, the smoothed point cloud, and the reconstructed mesh of the point cloud, respectively. Bottom show the fine reconstruction in different views.

reconstruction of dynamic objects, making them dense, coherent, and complete, see Fig. 1 for instance.

The experimental setup of our work consists of calibrated 2D (Point Grey Flea 2 Colour Camera) and 3D (Velodyne LiDAR HDL-64E) cameras attached to a moving vehicle. Given multiple sparse and partial point clouds of a rigidly moving object, observed from different view ports, we aim to obtain its high quality reconstruction by exploiting both $2 \mathrm{D}$ and $3 \mathrm{D}$ observation. This paper harnesses the achievements of [4] on detecting dynamic objects. In [4], dynamic objects are reconstructed by registering the sparse point clouds with the help of Random Sample Consensus (RANSAC) on 3D-3D correspondences for this problem. Final results of [4] are noisy, multi-layered and very often incomplete. We argue that such reconstruction is not particularly suitable for the sparse point clouds, since there exists no precise 3D-3D correspondence.

The main contributions of this paper are two-folded: (1) A complete pipeline for high quality 3D reconstruction of dynamic objects using 2D-3D camera setup attached to a moving vehicle has been proposed; (2) Our formulation leverages from the success of closest-point and consensus based methods, while complementing each other in their unfavourable conditions. The proposed optimization framework is robust as well as accurate. 


\section{LITERATURE REVIEW}

Recent studies can be categorized as: Iterative Closest Point (ICP) -based point cloud alignment [7-14], RANSAC-based $[2,4,15,16]$ point cloud registration, and volumetric representation -based point cloud fusion [17-19]. ICP-based methods are robust and accurate in general. Yet, it can easily fail when point cloud's geometric structure is low, which yields to an ill-posed problem. RANSAC-based approaches are robust and efficient but require sufficient number of precise 3D3D matching pairs. Volumetric representation -based algorithms utilize the Signed Distance Function to describe the object surface using RGB-D camera. Volumetric representation methods work nicely for dense point cloud registration of large scene, while they suffer over-smoothing problems.

\section{ROBUST POINT CLOUDS REGISTRATION}

To register a sequence of sparse point clouds in a common coordinate frame, we formulate an optimization problem supported by their noisy motion trajectories. An accurate registration is the key for obtaining high quality textured surface reconstruction of the dynamic objects.

\subsection{Liniarized Rigid Motion Formulation}

Given a set of correspondences between two 3D point clouds, the exact solution for rigid motion parameters, i.e. $\mathrm{R}$ and $\mathrm{t}$, can be obtained in a linear manner. Let $\mathbf{X}=[x, y, z]^{\top}$ and $\mathrm{Y}=\left[x^{\prime}, y^{\prime}, z^{\prime}\right]^{\top}$ be two corresponding 3D points under rigid transformation, denoted as $\mathrm{X}=\mathrm{RY}+\mathrm{t}$. In which, $\mathrm{R}$ is the $3 \times 3$ rotation matrix and $t$ is the $3 \times 1$ translation matrix. By employing the Gibbs representation [20] and the Cayley transform [21], the 3D registration problem can be formulated as a linear system [4]:

$$
\left[\begin{array}{l}
x-x^{\prime} \\
y-y^{\prime} \\
z-z^{\prime}
\end{array}\right]=\left[\begin{array}{cccccc}
0 & z+z^{\prime} & -\left(y+y^{\prime}\right) & 1 & 0 & 0 \\
-\left(z+z^{\prime}\right) & 0 & \left(x+x^{\prime}\right) & 0 & 1 & 0 \\
y+y^{\prime} & -\left(x+x^{\prime}\right) & 0 & 0 & 0 & 1
\end{array}\right]\left[\begin{array}{c}
g_{x} \\
g_{y} \\
g_{z} \\
\tilde{t}_{x} \\
\tilde{t}_{y} \\
\tilde{t}_{z}
\end{array}\right]
$$

where $\left[g_{x}, g_{y}, g_{z}\right]^{\top}$ are the three rotation elements of Gibbs rotation representation. $\left[\tilde{t}_{x}, \tilde{t}_{y}, \tilde{t}_{z}\right]^{\top}=\left(\mathrm{I}_{3}+\mathrm{G}\right) \mathrm{t}$, in which $\mathrm{I}_{3}$ is the $3 \times 3$ identity matrix and $\mathrm{G}$ is the skew-symmetric form of Gibbs rotation angles. Since each matching pair provides 2 independent equations, solving the 6 unknown Eq. (1) requires minimum 3 pairs of correspondences. Moreover, a Random Sample Consensus (RANSAC) framework is adopted for the robustness toward outliers. In the presence of inaccurate correspondences, obtained from noisy motion trajectories, the quality of RANSAC registration is usually not very satisfactory. Therefore, this is further refined by minimizing the dual-weighted closet-point energy.

\subsection{Robust Closest-Point Energy Minimization}

When two overlapping point clouds of the same rigid object are given, the transformation between them is generally ob- tained by minimizing the energy derived from the closestpoints distance. In most of the cases, this energy is minimized using an iterative method - also known as Iterative Closest Point (ICP) algorithm [10,11]. In every step, the ICP algorithm considers closest points across two point clouds, say reference and model, to be the corresponding ones. Let $\mathbf{X}=\left\{\mathbf{X}_{1}, \ldots, \mathbf{X}_{n}\right\}$ be the reference point cloud, and $\mathbf{Y}=$ $\left\{\mathrm{Y}_{1}, \ldots, \mathrm{Y}_{m}\right\}$ be the new model, the robust method of ICP iteratively minimizes the following energy:

$$
\mathcal{E}_{I}(\hat{\mathrm{T}})=\min _{\mathrm{T}} \sum_{i=1}^{n} \rho\left(\min _{j \in\{1, \cdots, m\}}\left\|\mathrm{X}_{i}-\mathrm{TY}_{j}\right\|_{2}\right)
$$

where $\hat{T}$ is the desired transformation matrix that relates two point clouds. Note that the energy term $\mathcal{E}_{I}$ includes a robust cost function to handle noisy and partial data. Our choice of robust cost, say $\rho(x)$, is the Tukey's biweight function [22]:

$$
\rho(x)=\left\{\begin{array}{cc}
\left(\tau^{2} / 6\right)\left(1-\left[1-(x / \tau)^{2}\right]^{3}\right) & \text { if }|x| \leq \tau \\
\left(\tau^{2} / 6\right) & \text { if }|x|>\tau
\end{array},\right.
$$

and the weight of each corresponding pair is defined by:

$$
w(x)=\frac{1}{x} \frac{\mathrm{d} \rho(x)}{\mathrm{d} x}=\left\{\begin{array}{cc}
{\left[1-(x / \tau)^{2}\right]^{2}} & \text { if }|x| \leq \tau \\
0 & \text { if }|x|>\tau
\end{array}\right.
$$

where $\tau$ is the inlier threshold, such that outliers $(|x|>\tau)$ are assigned zero weights.

\subsection{Modified Closest-Point Energy Minimization}

While consensus-based registration method requires a subset of accurate correspondences, closest-point-based method requires rich structure of the point clouds. This prohibits us to make a choice of one method over another. Therefore, we propose to minimize a combined energy function - one from consensus, say $\mathcal{E}_{R}$, and the other from closest-point, say $\mathcal{E}_{I}$. We minimize the energy function in an iterative manner, hence use the terminology Dual-Weighted Iterative Closest Point (DW-ICP) for this method.

First, we define an energy function that measures the quality of the inlier set obtained form 3-point RANSAC. Note that due to the sparsity and noisy points, the inlier set obtained from RANSAC is not precise. Let $\left\{\mathrm{X}_{i} \leftrightarrow \mathrm{Y}_{i}\right\}, i=1, \ldots, k$ be the inlier set, the energy $\mathcal{E}_{R}$ for matching consensus can be expressed as:

$$
\mathcal{E}_{R}(\hat{\mathrm{T}})=\min _{\mathrm{T}} \sum_{i=1}^{k} \tilde{\rho}\left(\left\|\mathrm{X}_{i}-\mathrm{TY}_{i}\right\|_{2}\right),
$$

where $k \leq m, n$, and $\tilde{\rho}(\cdot)$ is the Huber's weight function:

$$
\begin{gathered}
\tilde{\rho}(x)=\left\{\begin{array}{cc}
\left(x^{2} / 2\right) & \text { if }|x| \leq \tilde{\tau} \\
\tilde{\tau}[|x|-(\tilde{\tau} / 2)] & \text { if }|x|>\tilde{\tau}
\end{array}\right. \\
\tilde{w}(x)=\frac{1}{x} \frac{\mathrm{d} \tilde{\rho}(x)}{\mathrm{d} x}=\left\{\begin{array}{cc}
1 & \text { if }|x| \leq \tilde{\tau} \\
(\tilde{\tau} /|x|) & \text { if }|x|>\tilde{\tau}
\end{array}\right.
\end{gathered}
$$




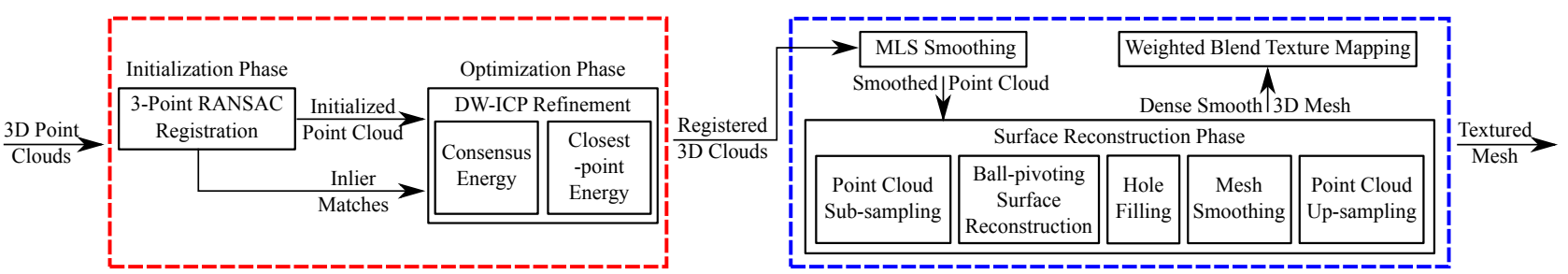

Fig. 2: Framework for High Quality Rigid Object Reconstruction: the Point Clouds Registration and Mesh Reconstruction.

where $\tilde{\tau}$ is the threshold for inlier matches. The Huber loss function is selected under the assumption that the provided inlier set is noisy with no severe outlier that needs to be completely discarded. In the spirit of Eq. (2) and Eq. (5), we formulate our combined energy function as follows:

$$
\begin{array}{r}
\mathcal{E}(\hat{\mathrm{T}})=\min _{\hat{\mathrm{T}}}\left\{\alpha \sqrt{\frac{1}{n} \sum_{i=1}^{n} \rho\left(\min _{j \in\{1, \cdots, m\}}\left\|\mathrm{X}_{i}-\mathrm{TY}_{j}\right\|_{2}\right)}+\right. \\
\left.(1-\alpha) \sqrt{\frac{1}{k} \sum_{i=1}^{k} \tilde{\rho}\left(\left\|\mathrm{X}_{i}-\mathrm{TY}_{i}\right\|_{2}\right)}\right\},
\end{array}
$$

where $\alpha$ is the regularization term to control the influence of the $\mathcal{E}_{I}$ and $\mathcal{E}_{R}$ energy terms. Rather than optimizing the closest-point energy $\mathcal{E}_{I}$ or matching consensus energy $\mathcal{E}_{R}$ independently, the DW-ICP aims to iteratively and simultaneously optimize the joint energy $\mathcal{E}$ of Eq. (8).

\section{3D RECONSTRUCTION FRAMEWORK}

A complete pipeline for high quality 3D reconstruction of rigidly moving objects, using 2D-3D camera setup attached to a moving vehicle, is shown in Fig. 2. There are two major steps involved, namely the Point Clouds Registration and Smooth Mesh Reconstruction.

\subsection{Point Cloud Registration}

Our method takes the 3D motion trajectories of a sequence of segmented point clouds obtained from [4] as input. First, we use the 3-Point RANSAC registration to roughly register the point clouds as initialization. Afterwards, the DW-ICP is applied to refine the registration. Note that (also refer to Eq. (8)) the DW-ICP iteratively minimizes a combined energy term, one from consensus $\mathcal{E}_{R}$ and other from closest-point $\mathcal{E}_{I}$, during the optimization process. On one hand, $\mathcal{E}_{I}$ minimizes the overall registration error of the whole 3D point clouds. On the other hand, $\mathcal{E}_{R}$ minimizes the registration error of the inliers obtained form RANSAC. These two terms are usually complementary to each other, which is the key to the success of the proposed optimization framework.

On top of traditional ICP, there are two main advantages of DW-ICP: (a) Feature matching constraint promises a proper registration regardless the poor geometry structure of the point clouds. (b) Robust estimation framework is preserved such that the algorithm is generic and robust to outliers during a long term registration.

\subsection{Mesh Reconstruction}

To reconstruct a photo-realistic high quality 3D model, a full pipeline is presented in Fig. 2 (blue box). There are three major steps involved, namely Moving Least Square (MLS) [23] point cloud smoothing, 3D Mesh Reconstruction, and Weighted Blend Texture Mapping [24].

Point Cloud Smoothing Due to the measurement noise of the laser scanner and imperfect registrations, a long sequenced registered point cloud consists of outliers and multilayer effects. Surface reconstructed from such point cloud suffers from many visual artefacts, such as spiky mesh and holes. Thus, a MLS algorithm, which smooths an unorganized point could using a polynomial fitting, is applied.

Surface Reconstruction Prior to the surface reconstruction, a sub-sampling processing according to the points' poisson-disk distribution [25] is applied. This avoids the repetition of redundant points (overlapped points) due to the multiple observations of the same scene. Later, a Ball Pivoting Triangulation algorithm [26] is used to establish the neighbour-points relationships, followed by a dilation operation for hole filling. Next, a Taubin Surface Smoothing method is adopted to smooth the reconstructed surface while preserving the sharp edges. Finally, a Least Square Subdivision approach [27] is performed to up-sample followed by re-meshing the point cloud to produce high quality meshes.

Texture Mapping We make use of 2D images acquired by the 2D-3D camera setup for texture mapping. During this process, photographic alignment between 3D mesh and images are required. Since the 2D-3D camera setup is already calibrated, and the motion of the camera is known, all the images are aligned with respect to the mesh reconstructed frame. The camera poses (between the camera and the reconstructed mesh) are estimated by computing the inverse of the transformation matrices (obtained from registration) and using the camera calibration parameters. Furthermore, the blurring during the texture fusion from multiple images is reduced by using a Weighted Blending algorithm.

\section{EXPERIMENTS}

For the evaluation, experiments were conducted on both synthetic and real (KITTI [1]) datasets. We generated three sets of synthetic data to quantify the robustness and accuracy of the proposed algorithms. Qualitative results of the proposed framework is presented using real data. In all of our ex- 

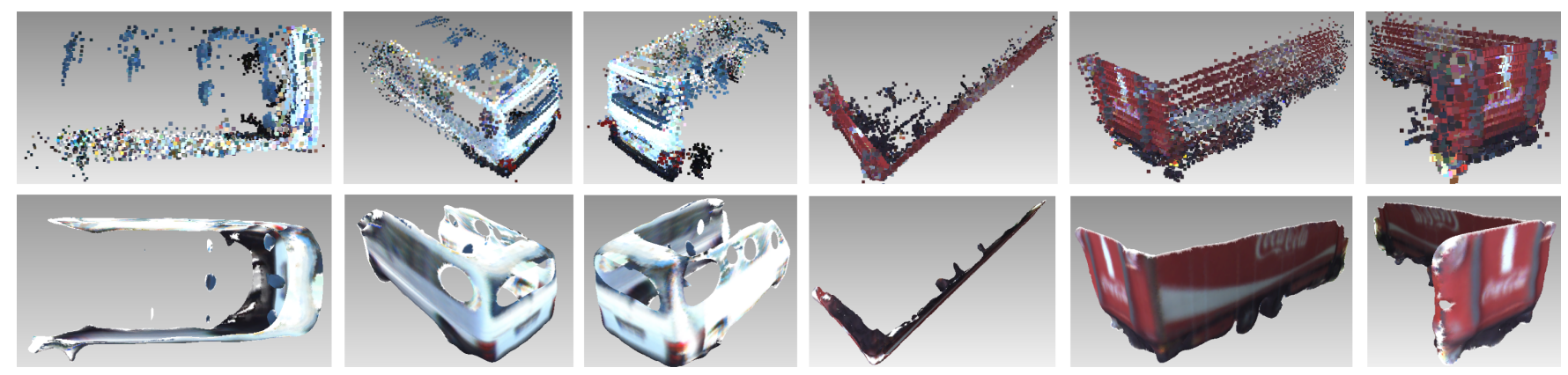

Fig. 3: Reconstructed Van and Cola Truck: top are registered point clouds using [4]; bottom are our high quality meshes.

periments, the algorithm parameters were set as: $\alpha=0.8$, $\tau=0.08 m, \tilde{\tau}=0.03 m$. The stopping conditions of the DWICP optimization are: rotation tolerance $\epsilon_{\mathrm{R}}=10 e-6$, translation tolerance $\epsilon_{\mathrm{T}}=10 e-6$, and maximum DW-ICP iteration as 100. All the experiments are conducted in a computer with Intel Quad Core i7-2640M, 2.80GHz, 8GB Memory.



Fig. 4: Synthetic Trajectory of Van Object.

Synthetic Datasets: The synthetic datasets were generated for three objects from KITTI dataset, namely Van, Red Car, and Cola Truck. An example of synthetic trajectory and visible parts are shown in Fig. 4. Note that some frames in the trajectory consist of different views (sides or back views), resembling the partial overlapping and poor 3D geometric structure problems. To evaluate the robustness of the algorithm, different levels of Gaussian noise were added to the model. We applied 10 different levels of noise, from 0.005 to 0.050 in meters. The maximum noise level is chosen as 2.5 times higher than the expected accuracy $(0.02 \mathrm{~m})$ of the Velodyne laser scanner.

Figure 5 shows the performances of 4 different algorithms, namely 3-Point RANSAC [4], 3-Point RANSAC + ICP refinement [10], 3-Point RANSAC + Robust-ICP [13] and 3-Point RANSAC + DW-ICP. The overall performance of the algorithms are (top-down) ranked as: DW-ICP, RobustICP, RANSAC+ICP and RANSAC. The Robust-ICP (using M-Estimator) has significantly better performance against that of traditional ICP. Most importantly, the proposed DWICP constantly outperforms the other approaches, regardless of rotation and translation.

Real Datasets: Table 1 depicts the dataset information, where the 3D Error (averaged Leave-One-Out Error) metric is used to quantify the registration performance. The registration error of our method is consistently lower than [4],


Fig. 5: Synthetic Data Quantification: top and bottom are averaged translation and rotation errors on Van, Red Car, and Cola Truck dataset, respectively.

although we have slightly more computation time due to the DW-ICP refinement process. Moreover, the high quality reconstructions of Fig. 1 and Fig. 3 are obtained using the proposed framework of Fig. 2. Note that the objects are reconstructed from long-term and faraway observations (see column Dist. of Table 1), under the situations that both target objects and camera system are moving in high speeds. The framework effectively overcomes the accumulation errors during the registration process and products very satisfactory results. Figure 3 demonstrates that significantly better results of our method are achieved comparing to [4].

\begin{tabular}{|c|c|c|c|c|c|c|c|}
\hline \multirow{2}{*}{ Object } & \multirow{2}{*}{ \# Frame } & \multirow{2}{*}{ Sides } & \multirow{2}{*}{$\begin{array}{l}\text { Dist. } \\
\text { (m) }\end{array}$} & \multicolumn{2}{|c|}{ 3-Point RANSAC [4] } & \multicolumn{2}{|c|}{ Ours } \\
\hline & & & & Error $(\mathrm{m})$ & Time (s) & Error (m) & Time (s) \\
\hline Van & 44 & 3 & 16.5 & 0.0150 & 3.1 & 0.0131 & 4.6 \\
\hline Red Car & 60 & 3 & 10.8 & 0.0084 & 2.8 & 0.0080 & 4.3 \\
\hline Cola Truck & 48 & 2 & 30.0 & 0.0234 & 3.7 & 0.0229 & 4.1 \\
\hline
\end{tabular}

Table 1: Dataset Information: Col. Sides is number of object sides (left, right, back, and front) being captured. Col. Dist. is the averaged distance from the camera to the object. Col. 3Point RANSAC [4] and Col. Ours show their respective averaged $3 \mathrm{D}$ error and computation time.

\section{CONCLUSION}

We have proposed an effective optimization method, which combines the idea of consensus and closest-point in a common framework, to register highly sparse 3D point clouds from long term observations. Furthermore, We present a complete pipeline for high quality 3D mesh reconstruction. Results obtained from several experiments on both synthetic and real data were very satisfactory. 


\section{REFERENCES}

[1] Andreas Geiger, Philip Lenz, and Raquel Urtasun, "Are we ready for autonomous driving? the kitti vision benchmark suite," in Computer Vision and Pattern Recognition (CVPR), 2012 IEEE Conference on. IEEE, 2012, pp. 3354-3361.

[2] Danda Pani Paudel, Cédric Demonceaux, Adlane Habed, Pascal Vasseur, and In So Kweon, "2d-3d camera fusion for visual odometry in outdoor environments," in 2014 IEEE/RSJ International Conference on Intelligent Robots and Systems. IEEE, 2014, pp. 157-162.

[3] Ji Zhang and Sanjiv Singh, "Visual-lidar odometry and mapping: Low-drift, robust, and fast," in Robotics and Automation (ICRA), 2015 IEEE International Conference on. IEEE, 2015, pp. 2174-2181.

[4] Cansen Jiang, Danda Pani Paudel, Yohan Fougerolle, David Fofi, and Cédric Demonceaux, "Static-map and dynamic object reconstruction in outdoor scenes using 3-d motion segmentation," IEEE Robotics and Automation Letters, vol. 1, no. 1, pp. 324-331, 2016.

[5] Cansen Jiang, Danda Pani Paudel, Yohan Fougerolle, David Fofi, and Cédric Demonceaux, "Reconstruction 3D de scènes dynamiques par segmentation au sens du mouvement," in Le 20èmé Congrès National sur la Reconnaissance des Formes et l'Intelligence Artificielle (RFIA), Clermont-Ferrand, France, June 2016.

[6] Cansen Jiang, Yohan Fougerolle, David Fofi, and Cédric Demonceaux, "Dynamic 3d scene reconstruction and enhancement," in 19th International Conference Image Analysis and Processing (ICIAP), Catania, Italy, Sept. 2017. Lecture Notes in Computer Science. Springer, Berlin, Heidelberg.

[7] Zhengyou Zhang, "Iterative point matching for registration of free-form curves and surfaces," International Journal of Computer Vision, vol. 13, no. 2, pp. 119-152, 1994.

[8] François Pomerleau, Francis Colas, Roland Siegwart, et al., "A review of point cloud registration algorithms for mobile robotics," Foundations and Trends $®$ in Robotics, vol. 4, no. 1, pp. 1-104, 2015.

[9] Mouna Attia, Yosr Slama, and Mohamed Amine Kamoun, "On performance evaluation of registration algorithms for $3 \mathrm{~d}$ point clouds," in Computer Graphics, Imaging and Visualization (CGiV), 2016 13th International Conference on. IEEE, 2016, pp. 45-50.

[10] Paul J Besl and Neil D McKay, "Method for registration of 3d shapes," in Robotics-DL tentative. International Society for Optics and Photonics, 1992, pp. 586-606.

[11] Yang Chen and Gérard Medioni, "Object modelling by registration of multiple range images," Image and vision computing, vol. 10, no. 3, pp. 145-155, 1992.

[12] Paul W Holland and Roy E Welsch, "Robust regression using iteratively reweighted least-squares," Communications in Statistics-theory and Methods, vol. 6, no. 9, pp. 813-827, 1977.

[13] Andrew W. Fitzgibbon, "Robust registration of $2 \mathrm{~d}$ and $3 \mathrm{~d}$ point sets," Image and Vision Computing, vol. 21, no. 13, pp. 11451153, 2003.
[14] Dennis Christie, Cansen Jiang, Danda Pani Paudel, and Cédric Demonceaux, "3d reconstruction of dynamic vehicles using sparse 3d-laser-scanner and 2d image fusion," in 2016 International Conference on Informatics and Computing (ICIC), Oct 2016, pp. 61-65.

[15] Chu-Song Chen, Yi-Ping Hung, and Jen-Bo Cheng, "Ransacbased darces: A new approach to fast automatic registration of partially overlapping range images," IEEE Transactions on Pattern Analysis and Machine Intelligence, vol. 21, no. 11, pp. 1229-1234, 1999.

[16] Dhanya S Pankaj and Rama Rao Nidamanuri, "A robust estimation technique for $3 \mathrm{~d}$ point cloud registration," Image Analysis \& Stereology, vol. 35, no. 1, pp. 15-28, 2016.

[17] Carl Yuheng Ren, Victor Prisacariu, David Murray, and Ian Reid, "Star3d: Simultaneous tracking and reconstruction of 3d objects using rgb-d data," in Proceedings of the IEEE International Conference on Computer Vision, 2013, pp. 1561-1568.

[18] Stefan May, Philipp Koch, Rainer Koch, Christian Merkl, Christian Pfitzner, and Andreas Nüchter, "A generalized 2d and 3d multi-sensor data integration approach based on signed distance functions for multi-modal robotic mapping.," in $V M V$, 2014, pp. 95-102.

[19] Michael Zollhöfer, Angela Dai, Matthias Innmann, Chenglei Wu, Marc Stamminger, Christian Theobalt, and Matthias Nießner, "Shading-based refinement on volumetric signed distance functions," ACM Transactions on Graphics (TOG), vol. 34, no. 4, pp. 96, 2015.

[20] Josiah Willard Gibbs, Elements of vector analysis: arranged for the use of students in physics, Tuttle, Morehouse \& Taylor, 1884.

[21] Hatem Alismail, L Douglas Baker, and Brett Browning, "Continuous trajectory estimation for $3 \mathrm{~d}$ slam from actuated lidar," in 2014 IEEE International Conference on Robotics and Automation (ICRA). IEEE, 2014, pp. 6096-6101.

[22] Noel Cressie and Douglas M Hawkins, "Robust estimation of the variogram: I," Journal of the International Association for Mathematical Geology, vol. 12, no. 2, pp. 115-125, 1980.

[23] Peter Lancaster and Kes Salkauskas, "Surfaces generated by moving least squares methods," Mathematics of computation, vol. 37, no. 155, pp. 141-158, 1981.

[24] Marco Callieri, Paolo Cignoni, Massimiliano Corsini, and Roberto Scopigno, "Masked photo blending: Mapping dense photographic data set on high-resolution sampled 3d models," Computers \& Graphics, vol. 32, no. 4, pp. 464-473, 2008.

[25] Massimiliano Corsini, Paolo Cignoni, and Roberto Scopigno, "Efficient and flexible sampling with blue noise properties of triangular meshes," IEEE Transactions on Visualization and Computer Graphics, vol. 18, no. 6, pp. 914-924, 2012.

[26] Fausto Bernardini, Joshua Mittleman, Holly Rushmeier, Cláudio Silva, and Gabriel Taubin, "The ball-pivoting algorithm for surface reconstruction," IEEE transactions on visualization and computer graphics, vol. 5, no. 4, pp. 349-359, 1999.

[27] Simon Boyé, Gael Guennebaud, and Christophe Schlick, "Least squares subdivision surfaces," in Computer Graphics Forum. Wiley Online Library, 2010, vol. 29, pp. 2021-2028. 